\title{
A Study of Transmission Control Method for Distributed Parameters Measurement in Large Factories and Storehouses
}

\author{
Shujing Su, ${ }^{1}$ Min Yi, ${ }^{2}$ Wei Ji, ${ }^{1}$ Qing He, ${ }^{1,2}$ and Xiufeng Xie ${ }^{2}$ \\ ${ }^{1}$ National Key Laboratory of the Electronic Measurement Technology, North University of China, Taiyuan 030051, China \\ ${ }^{2}$ Key Laboratory of Instrumentation Science \& Dynamic Measurement, North University of China, Taiyuan 030051, China \\ Correspondence should be addressed to Shujing Su; sushujing@nuc.edu.cn
}

Received 6 October 2014; Accepted 20 February 2015

Academic Editor: George S. Tombras

Copyright (c) 2015 Shujing Su et al. This is an open access article distributed under the Creative Commons Attribution License, which permits unrestricted use, distribution, and reproduction in any medium, provided the original work is properly cited.

\begin{abstract}
For the characteristics of parameters dispersion in large factories, storehouses, and other applications, a distributed parameter measurement system is designed that is based on the ring network. The structure of the system and the circuit design of the master-slave node are described briefly. The basic protocol architecture about transmission communication is introduced, and then this paper comes up with two kinds of distributed transmission control methods. Finally, the reliability, extendibility, and control characteristic of these two methods are tested through a series of experiments. Moreover, the measurement results are compared and discussed.
\end{abstract}

\section{Introduction}

The key to implement distributed parameters measurement is to choose a suitable interconnection network and an effective control method of data transmission. Although ring network and star structure are most widely used in the application of interconnected network structure, they express different features. In the distributed network of star structure, the efficiency of each node in the data transmission is low, and power consumption is also high [1], so there are a lot of limitations in the application of distributed parameter measurement. However, the ring network has many attractive properties such as simplicity, extendibility, low degree, and easiness of implementation. As a result, ring network is most widely used in many applications, including multiloop networks [2,3], chordal ring network [4-6], large-capacity power electronics system [7], and distributed loop computer networks [8]. Therefore, taking advantages of ring network that could keep the parameters of each node independent in the interactive data communications, the data transmission between nodes and distributed control can be done.

Distributed transmission control in the field of wireless communications [9] used the means of monitor and interrupt transmission, making use of the arbitration agreement of wireless communication, so there were the shortcomings of system power and space communications cross talk. In electric power systems [10] used the method of network resource sharing, and there is the disadvantage of large network dependence and scattered resources. In the application of tracking construction operation [11] was the use of server monitor transmission mode, with the defect of high cost and complex protocol. Furthermore, in the field of hybrid drive [12], there was the disadvantage of the complex signal processing. These acquisition and transmission control methods due to the weakness in power consumption, cost, protocol, and other aspects are not suitable for using in large factories, storehouses, and other occasions for the measurement and control of distributed parameter. Therefore in this paper, we study the transmission control method of distributed parameter measurement in these occasions. Firstly, the principle of the two methods of distributed transmission control is introduced, and on this basis a series of experiments are carried out to test the performance of the two methods. And the two methods are sequential transmission method (STM) and independent transmission method (ITM).

This paper is structured as follows: the next section depicts the distributed measurement system and its circuit structure. Afterward, Section 3 firstly describes the communication protocol frame and secondly proposes two kinds of transmission control methods; Section 4 presents 


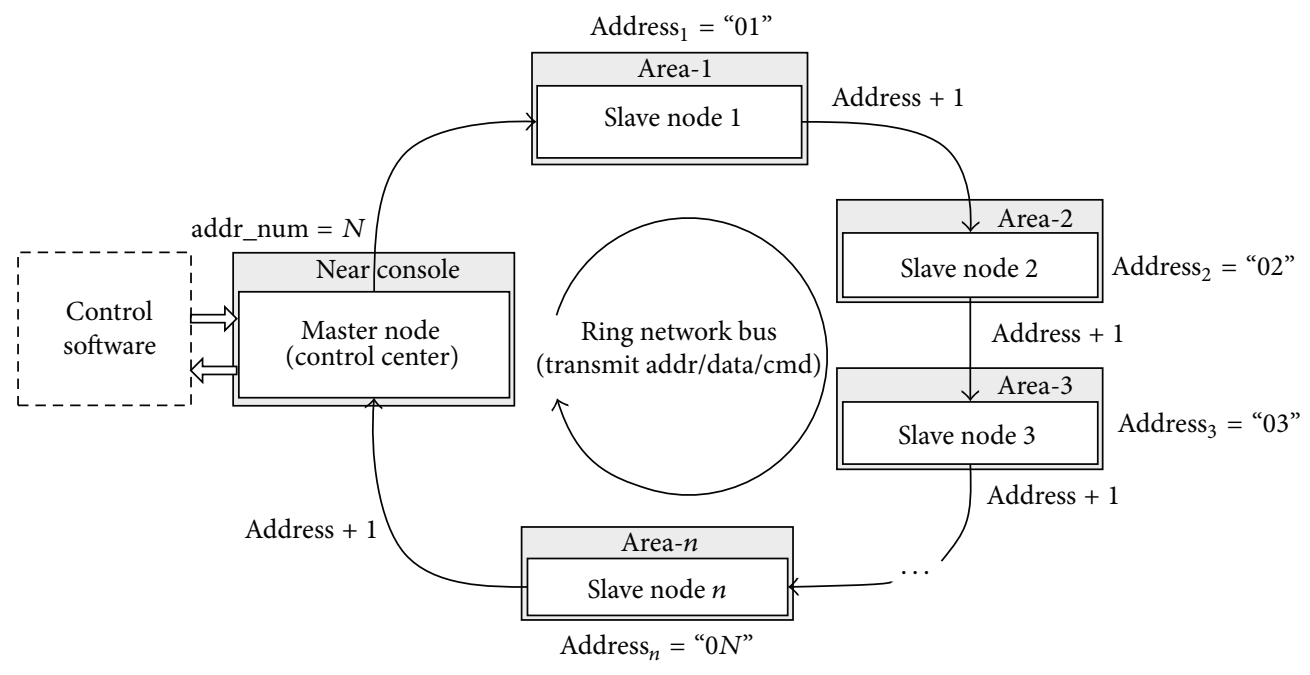

FIGURE 1: Structure of distributed multiparameter measurement system.

the experiments of control characteristic, experiment analysis, and discussion; finally, conclusions are given in Section 5.

\section{Distributed Measurement System and Circuit Structure}

2.1. Measurement System. Distributed multiparameter measurement system is shown in Figure 1. The ring network is composed of a master node, $n$ slave node, and the bus that connects these nodes. Slave nodes distributed in different regions are responsible for parameters measurement of their respective regions and the measured data transmission. The master node is the control center of the entire system and achieves the control of bus arbitration and centralized data processing by making use of the token ring protocol $[13,14]$. Under the control of master node, slave nodes' time-sharing gets the bus occupancy rights, so the bus is necessary to support high-speed data transmission. LVDS (low voltage differential signaling) is a high-speed data transmission and physical layer interface technology, and it has a large spread due to the high speed (up to $3 \mathrm{Gbps}$ ), low power consumption, low noise, and fast edge [15].

Before measurement, computer software through master node first completes address assignment and basic parameter configuration. As is shown in Figure 1, the control software sends the command of assignment address and the address 01 of slave node 1 . Then slave node 1 connecting to master node output interface receives the address 01 as its own address and saves it; meanwhile, this node send address 02 to slave node 2 . Then slave node 2 receives 02 as its address. By parity of reasoning, this working flow of address configuration can be done till the last node receives $0 N$ and sends $0 N+1$ to master node. And the master node saves the total number of nodes $($ addr_num $=0 N)$. So the master node is convenient to control these slave nodes by the assigned address in the process of data acquisition and transmission.
2.2. Node Circuit Structure. In a distributed measurement network, master node gets a lot of information sent by the computer control software, such as control, reset, start, and configuration parameters. The master node not only sends this information to all slave nodes and receives measured data, but also arbitrates the bus time that these nodes occupy. At last, the decoded and buffered data is transmitted to a computer for processing. Due to the different functions of master node and slave nodes, the circuit structures are different from each other. Further, the distance between two adjacent nodes may be far. So in order to compensate high-frequency loss and attenuation in long-distance data transmission [16], the data output interface of each node should add a high-speed differential buffer for cable driving and the data input interface should add an adaptive equalizer optimized for equalizing data.

The circuit structure of master node is shown in Figure 2, including a USB interface unit that communicates with a control computer [17-19], a control logic device, LVDS highspeed interface, the cable driving and equalizing circuit, and so forth. Programmable logic controller completes the system logic and timing control. Cable driver is used for preemphasis of the transmit signals and equalizer is used for compensation of signal loss through the long-distance transmission. LVDS interface realizes high-speed data communication.

As shown in Figure 3, circuit structure of a slave node includes sensor group, signal conditioning unit [20], analogto-digital conversion, the logic control unit, LVDS highspeed interface, and the cable driving and equalizing circuit. The sensor group accomplishes the measurement of multiple parameters in each area. Signal conditioning unit achieves signal amplification and wave filtering. Within a slave node occupying the bus time, this node sends measured data to the ring bus. When a node occupies the bus for data transmission, the remaining slave nodes receive and retransmit these data by the loopback FIFO. 


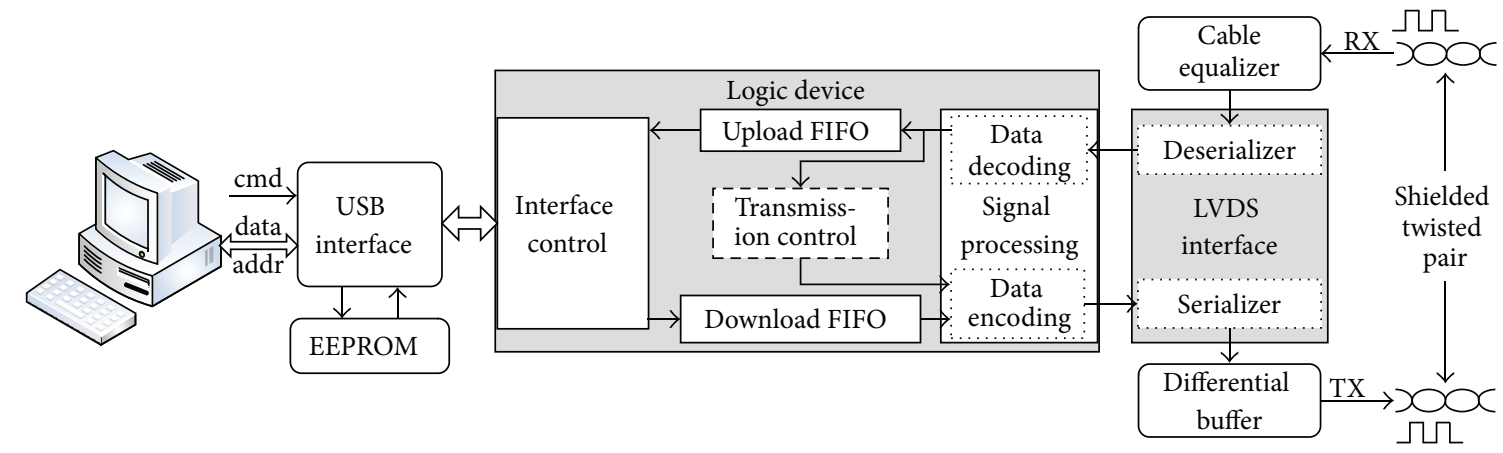

$\Omega 几$ Serial bit stream

Figure 2: The master node circuit.

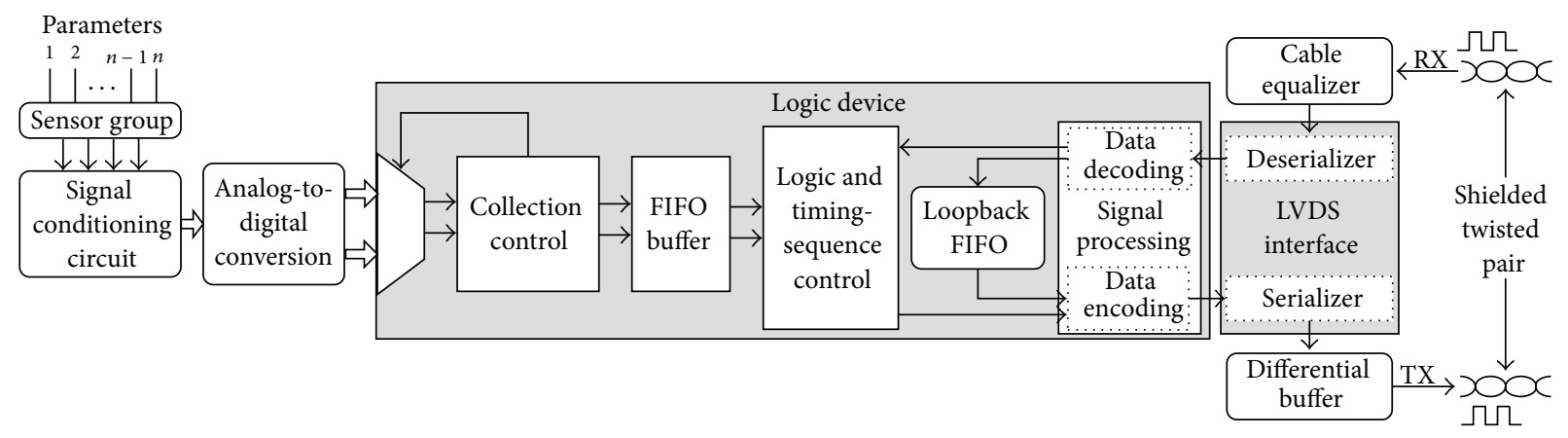

几几 Serial bit stream

FIgURE 3: The circuit composition of a slave node.

\section{Transmission Control Method}

Assuming the bit wide of instruction is $10 \mathrm{bit}$, then the high two bits (data[9:8]) are the symbol of data, address and command, and the remaining 8 bits (data[7:0]) are the instruction information, as shown in Table 1(a). When a slave node receives any information (data[9:0]), it should carry out identification and judgment. If data[9:8] is "00," this indicates that the received data[7:0] is the measurement data that a slave node transmits to the bus. If data[9:8] is " 01 ," data[7:0] is the command that the master node sends, and then this node performs the appropriate operation, such as data acquisition, transmission, and reset. If data[9:8] is "11," it means data[7:0] is the address of a slave node. A slave node receives the address information and judges whether the address is consistent with its identification address, and consistent slave node obtained the right of bus possession. When transmission command is received, the slave node according to the frame structure sends the measurement data to the bus. As is shown in Table 1(b), the data structure includes frame header, address of the slave node, data block, and the frame end. The size of the data block is decided by the number of acquisition channels and the sampling rate $f_{s}$.

If the number of acquisition channels is 16 , sampling rate is $f_{s}$, and word length is 16 bits. The data size $C$ collected by a slave node within $1 \mathrm{~ms}$ is regarded as the data block; then
TABLE 1: Transmission protocol structure.

(a) The representation of data/command/address

\begin{tabular}{lccccc}
\hline Type & Data[7:0] & Data & Command & $/$ & Address \\
\hline Flag bit & Data[9:8] & 00 & 01 & 10 & 11 \\
\hline
\end{tabular}

(b) Frame structure

\begin{tabular}{lccc}
\hline Frame header & Node address & Data block & Frame end \\
\hline EB 90 & Address $_{n}$ & Data $_{n}$ & Endcode \\
\hline
\end{tabular}

$C=16 \times 2 \times f_{s}$ bytes. And if the sampling rate $f_{s}=50 \mathrm{KSPS}$, then data block size $C=1.6 \mathrm{~K}$ bytes. Before transmission, these collected data should be buffered in the FIFO. In order to ensure that the data can be correctly buffered, FIFO buffer space is set as $4 \mathrm{~K}$ bytes.

When the master node controls data acquisition and transmission in different ways, it will result in different effects. So two kinds of transmission control methods are designed and studied in this paper, namely, STM and ITM. It is convenient to discuss their principle when the number of slave nodes is $n=3$. And the basic control signal is listed in Table 2. 
TABLE 2: Basic control signal.

\begin{tabular}{lccccccc}
\hline Signal & Address $_{1}$ & Address $_{2}$ & Address $_{3}$ & Endcode & start_acq & trans_order & stop_trans \\
\hline Data[9:8] & 11 & 11 & 11 & 01 & 01 & 01 & 01 \\
Data[7:0] & $0 \times 01$ & $0 \times 02$ & $0 \times 03$ & $0 \times 10$ & $0 \times 11$ & $0 \times 12$ & $0 \times 13$ \\
\hline
\end{tabular}

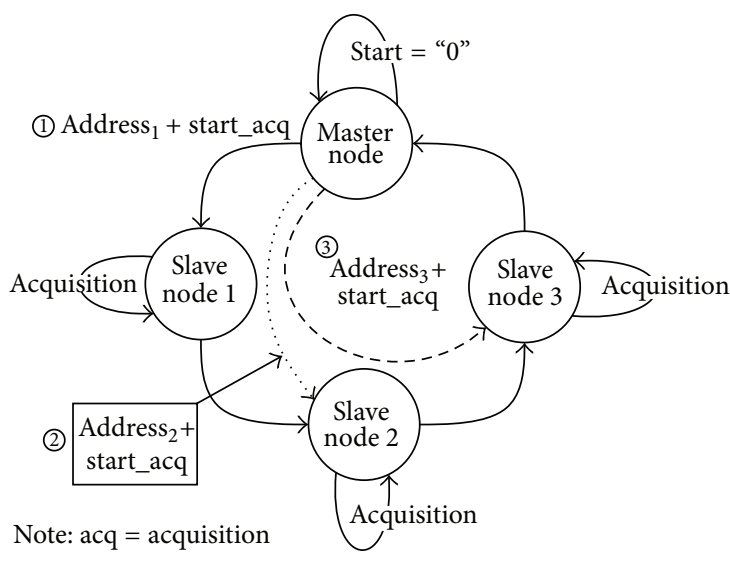

(a) The figure of state conversion of acquisition

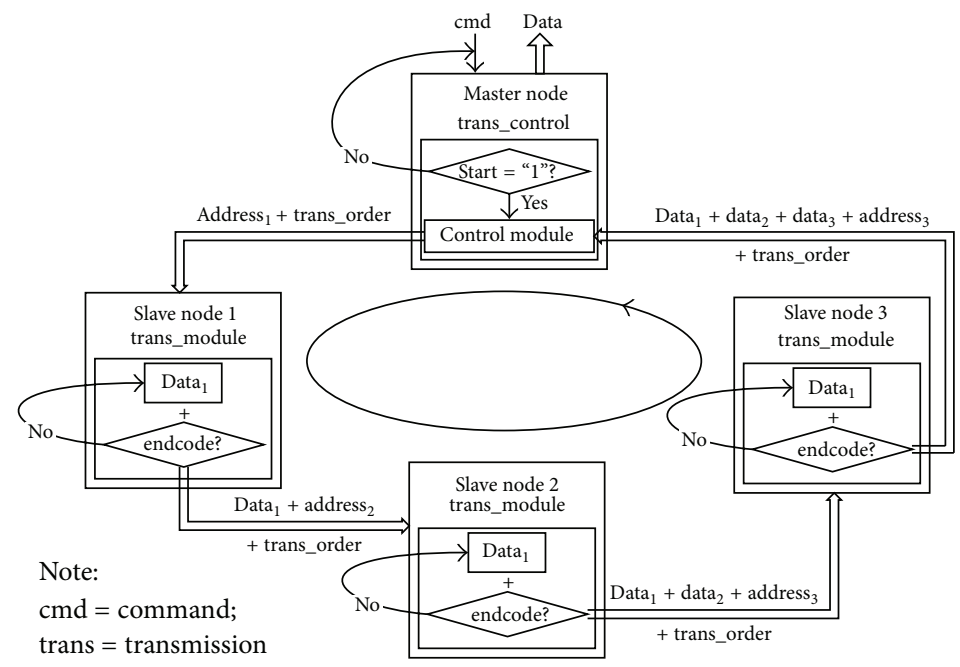

(b) The flowchart of data transmission

FIGURE 4: The flowchart of data acquisition and transmission in the STM when $n=3$.

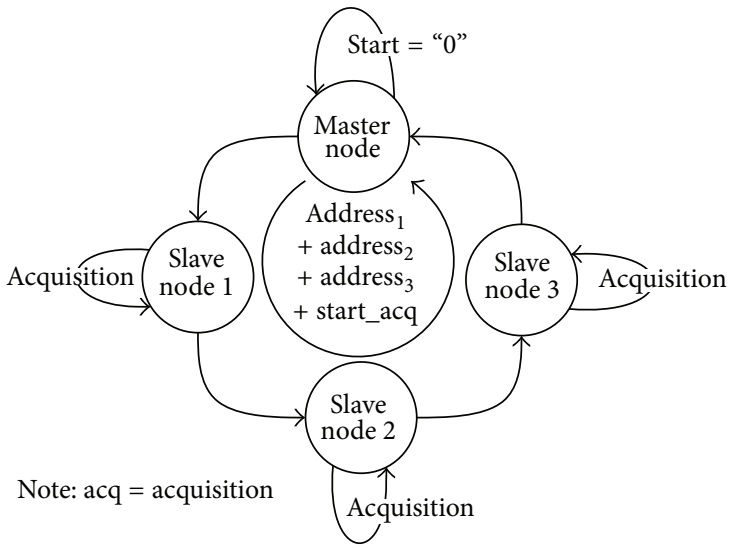

(a) The figure of state conversion of acquisition

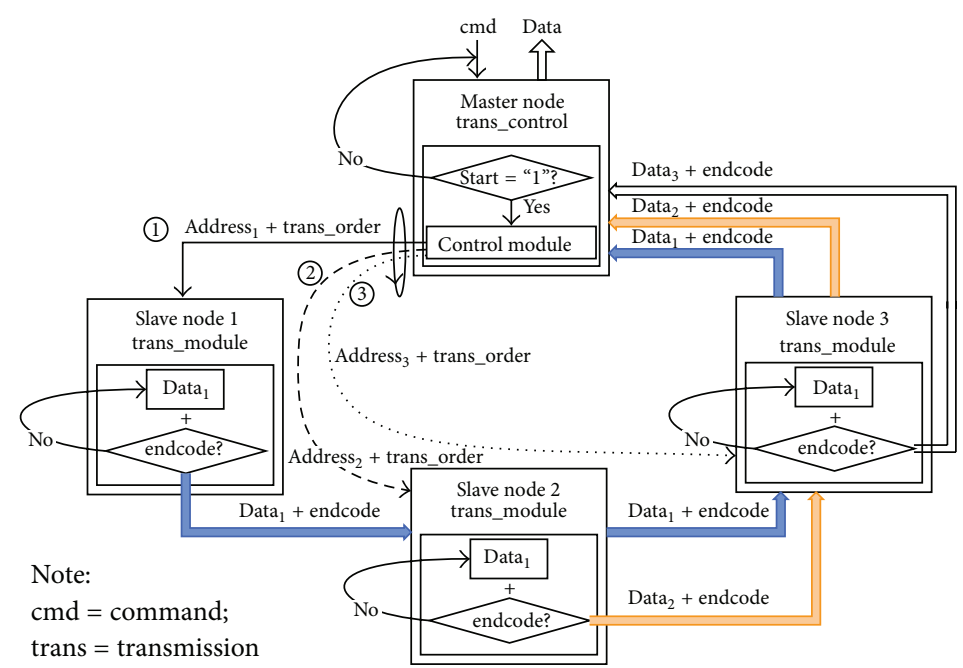

(b) The flowchart of data transmission

FIgURE 5: The flowchart of data acquisition and transmission in the ITM when $n=3$.

3.1. Sequential Transmission Method(STM). After address configuration is completed, master node sends the address and start acquisition command (start_acq) to slave nodes in order. Each salve node receives and forwards all the information that the master node sends. The state conversion of node acquisition and transmission control is shown in Figure 4. Meanwhile, the master node sends the address (address ${ }_{1}=$ 01) of slave node 1 and transmission command (trans_order) to the bus. Slave node 1 identifies the address ${ }_{1}$ and the transfer command and then gets the right of bus possession. When a frame data acquisition has completed, this node sends the data to the bus together with the next node address and the transmission command. So all the information that the next node receives is $\mathrm{EB}+90+$ address $_{1}+$ data $_{1}+$ endcode + address $_{2}+$ trans_order (illustration omits frame header), and node 1 releases this bus tenure.

After node 2 receives the information that the previous node transmitted to the bus, it transmits this information and 


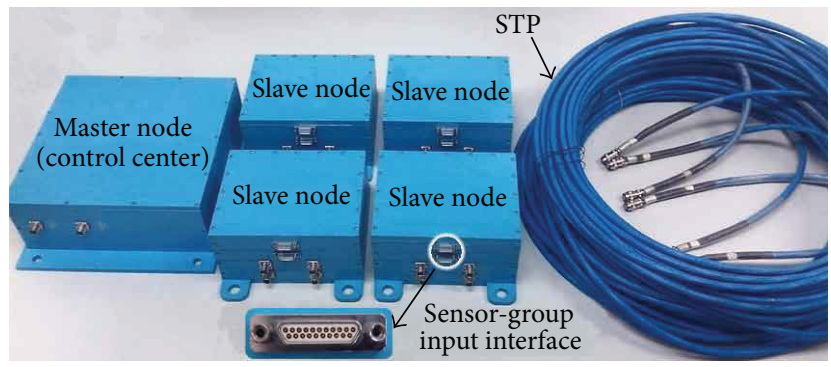

FIGURE 6: Experimental platform.

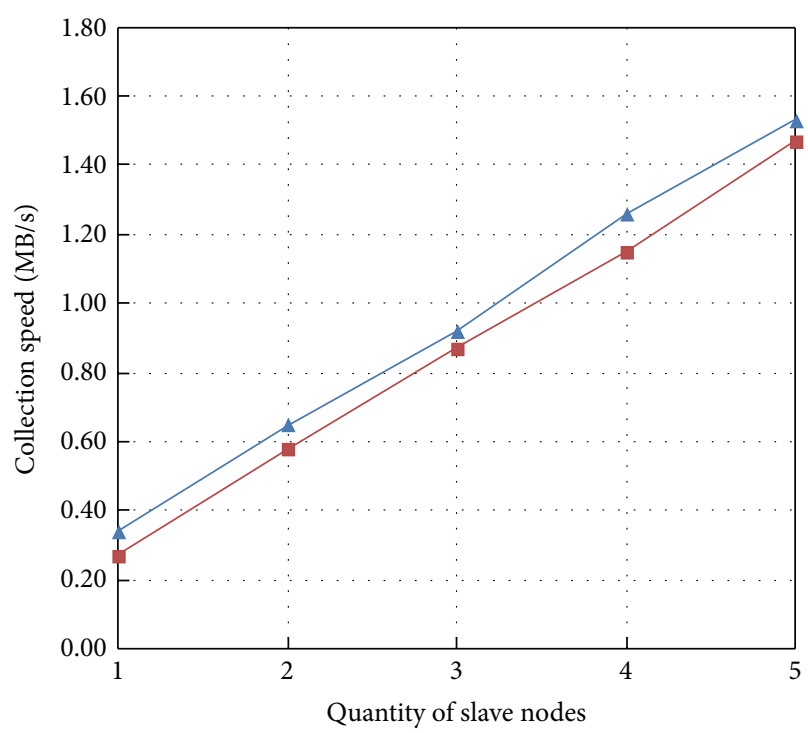

(a) Sampling rate $=10 \mathrm{KSPS}$

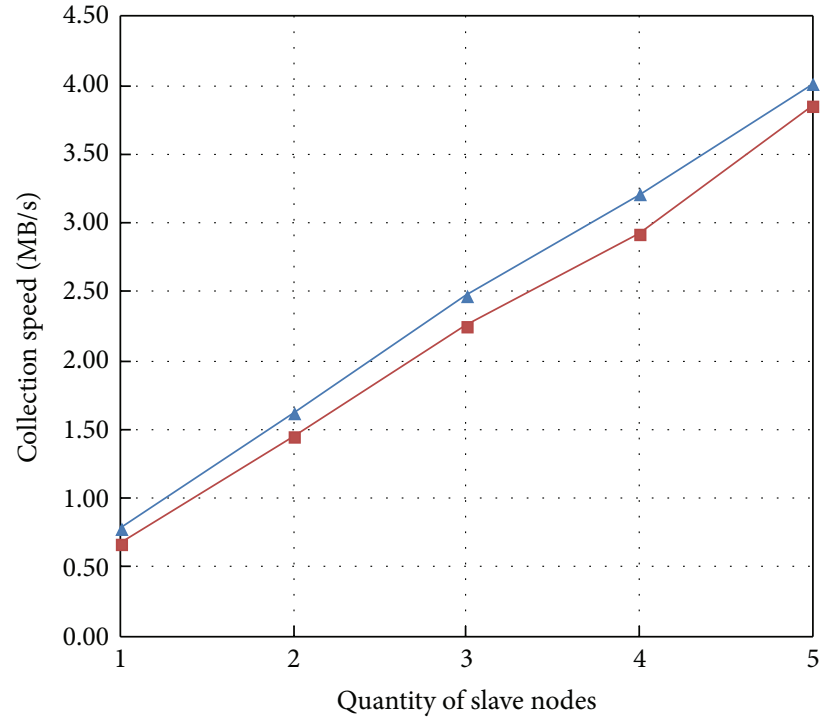

(b) Sampling rate $=25$ KSPS

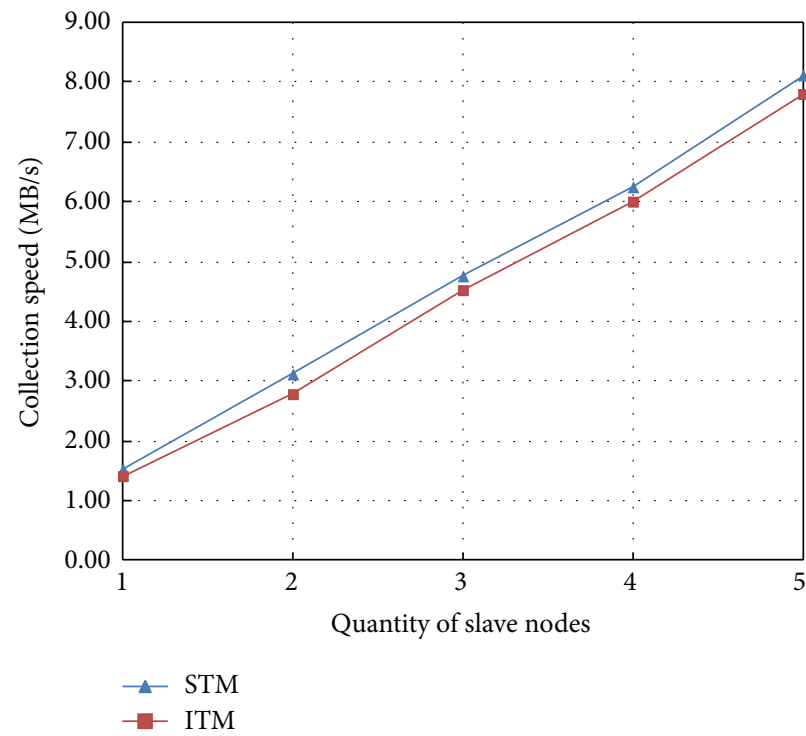

(c) Sampling rate $=50 \mathrm{KSPS}$

FIGURE 7: The relationship between acquisition speed and the number of slave nodes at different node sampling rate. 


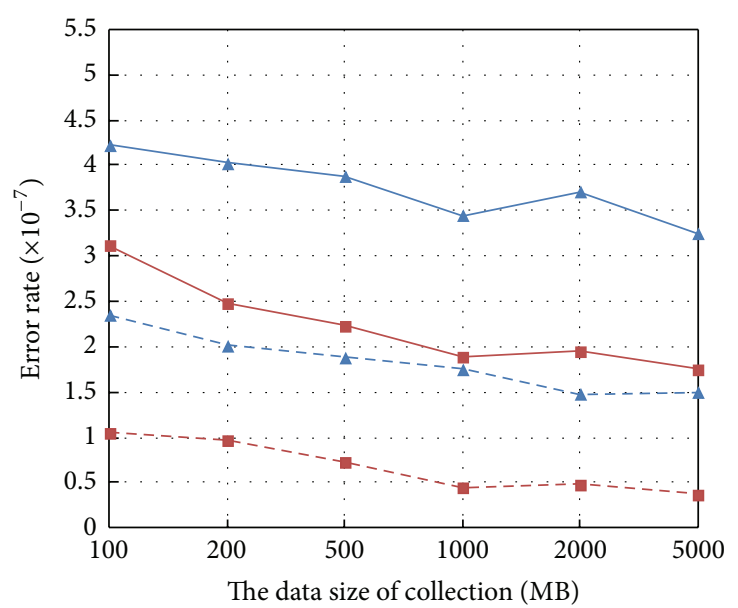

(a) STP length $=20 \mathrm{~m}$

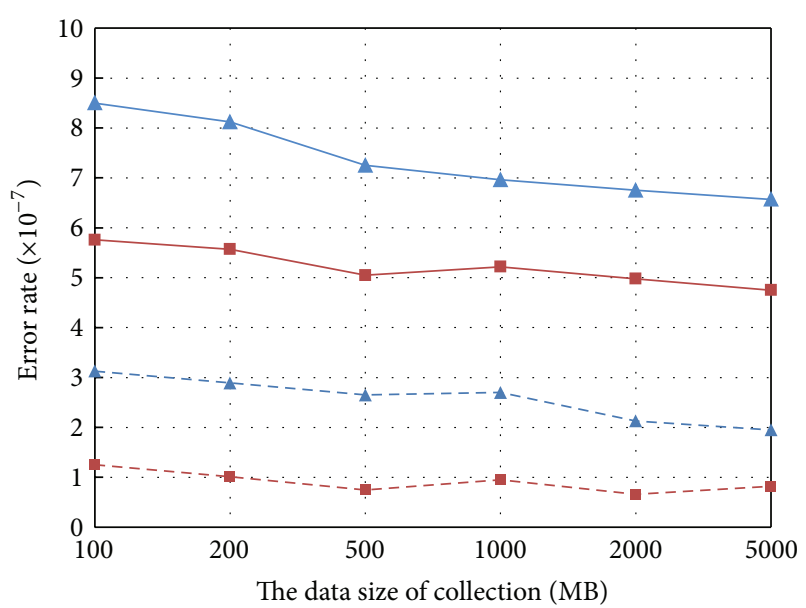

(b) STP length $=50 \mathrm{~m}$

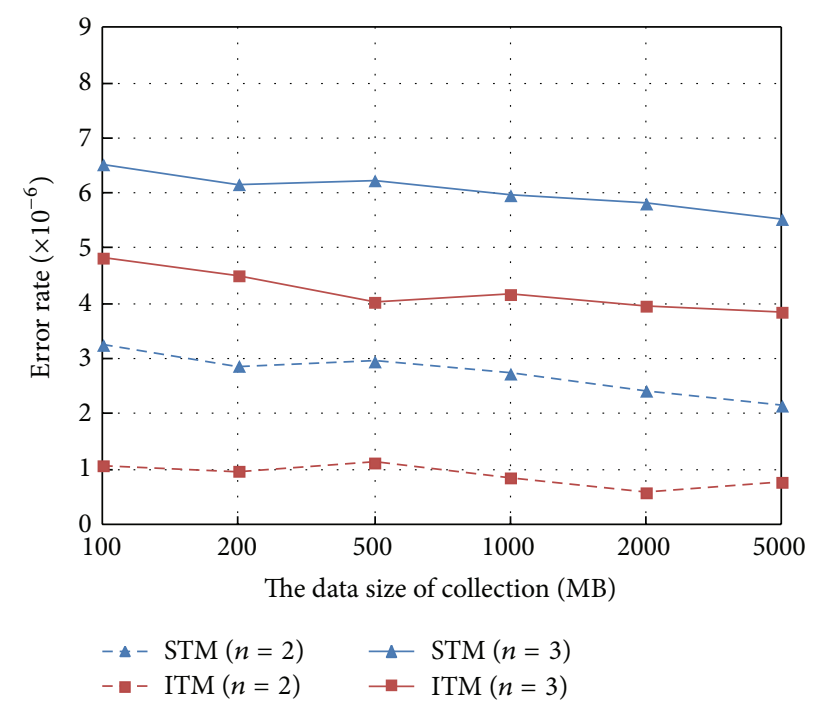

(c) STP length $=100 \mathrm{~m}$

FIGURE 8: The relationship between the data size and error rate when twisted length is $20 \mathrm{~m}$, $50 \mathrm{~m}$, or $100 \mathrm{~m}$.

gets the bus tenure. This node also adds the next node address and the transmission command in the end of the frame data. Other nodes perform similar operations. When master node receives $\mathrm{EB}+90+$ address $_{1}+$ data $_{1}+$ endcode $+\mathrm{EB}+90+$ address $_{2}+$ data $_{2}+$ endcode $+\cdots+\mathrm{EB}+90+$ address $_{n}+$ data $_{n}$ + endcode, it indicates the completion of a distributed data transmission process. Then the above process is restarted, and this method is called sequential transmission method. At last, received data which is buffered in the master node is transferred to a computer to be analyzed and processed.

3.2. Independent Transmission Method (ITM). As shown in Figure 5 (frame header is omitted), the master node first sends these nodes' addresses and the start acquisition command (start_acq) to all nodes. Secondly, every slave node receives and retransmits all the information sent by master node. After a node receives the matched address, it receives the acquisition command and begins data acquisition. Thirdly, the master node sends the address 01 and transmission command (trans_order) to the slave node 1 . And then this node receives the command and sends a frame data $\left(\mathrm{EB}+90+\right.$ address $_{1}+$ data $_{1}+$ endcode $)$ to the bus. At the same time, the other nodes on the bus use the internal forwarding FIFO to transmit all the information.

Furthermore, the master node receives the end flag (endcode) to judge that the data transmission is completed and then immediately sends the command to suspend transmission (stop_trans) to cancel the bus tenure of node 1 and starts transmission control of the next node. Finally, the master node receives the data of node $n\left(\mathrm{~EB}+90+\right.$ address $_{n}$ + data $_{n}+$ endcode) and suspends its transmission. Then the next distributed transmission process is restarted according to the above steps, so this method is named independent transmission method. 


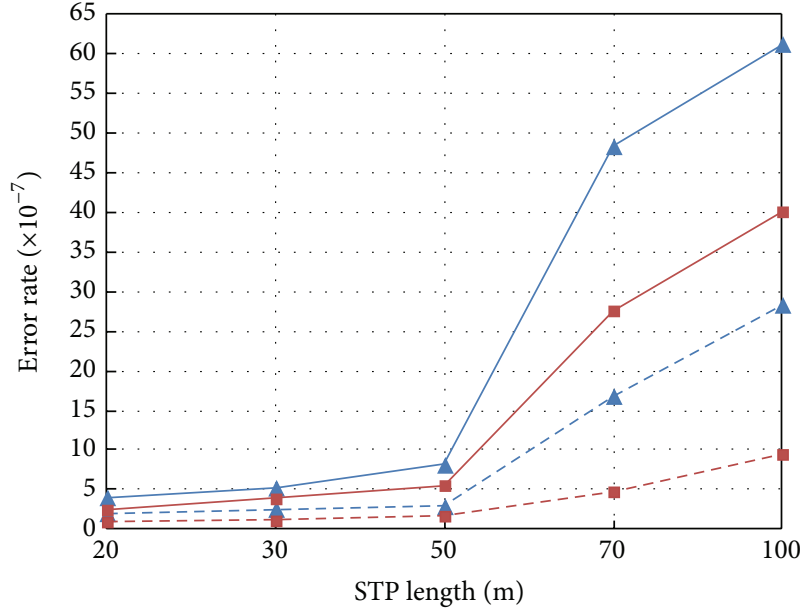

(a) The data size $=200 \mathrm{MB}$

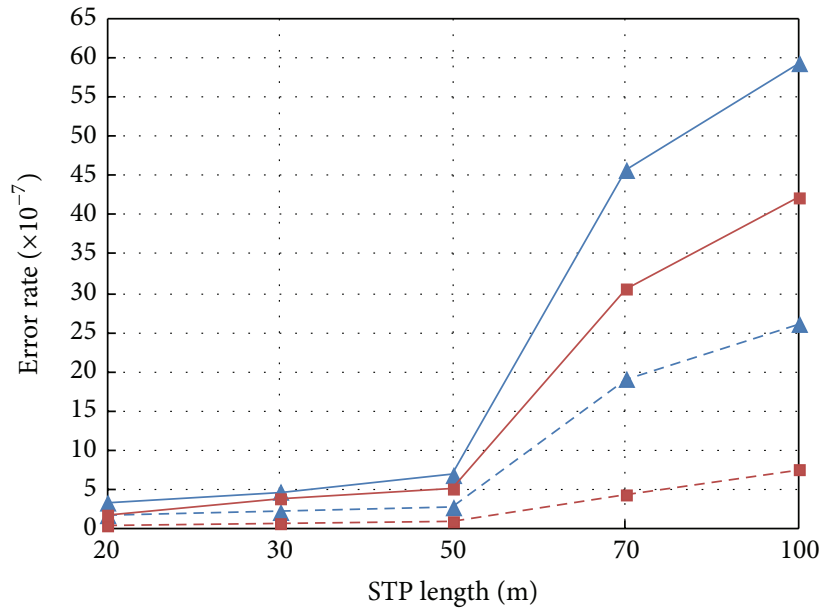

(b) The data size $=1000 \mathrm{MB}$

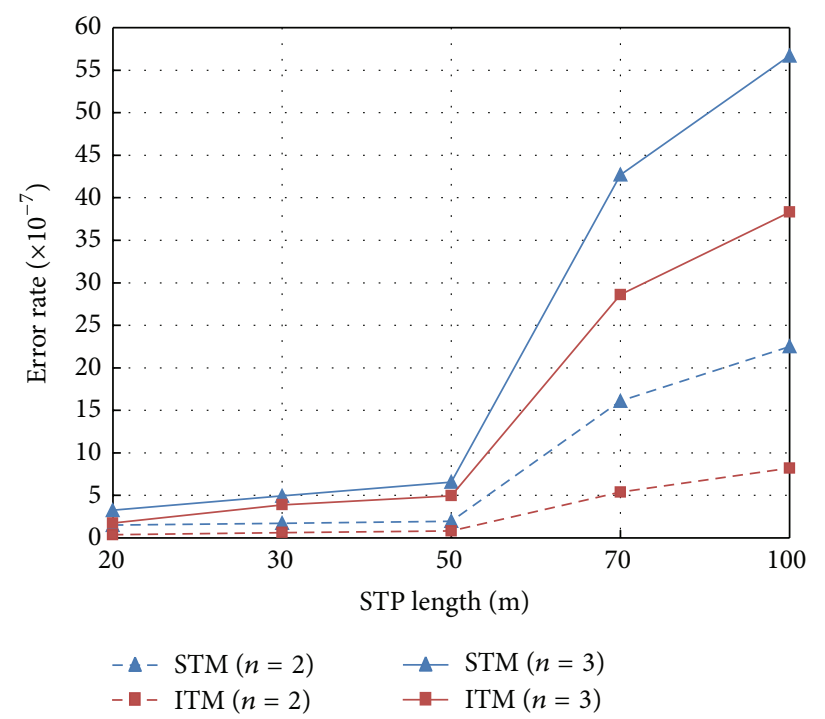

(c) The data size $=5000 \mathrm{MB}$

FIgUre 9: The error rate as a function of the STP length when data size is $200 \mathrm{MB}, 1000 \mathrm{MB}$, or $5000 \mathrm{MB}$.

\section{Transmission Control Experiment}

4.1. The Structure of Experiment System. To test the transmission control characteristics of the two methods, we establish an experimental hardware platform which is shown in Figure 6. In the platform, LVDS high-speed serializer/ deserializer, cable driver, and equalizer select National Semiconductor's DS92LV18, DS15BA101, and DS15EA101 [21, 22]. The timing and logic control unit choose the Xilinx Spartan$3 \mathrm{E}$ series FPGA, and the connection between nodes uses the category 6 (CAT6) shielded twisted pair (STP) cable. During the experiment, the transmission frequency of the master node and slave nodes is $30 \mathrm{MHz}$; namely, the transmission rate is $300 \mathrm{Mbps}$.

\subsection{Experiment Result and Discussion}

Experiment I. First of all, the master node connects one slave node and sets the node sampling rate to $10 \mathrm{KSPS}$. The slave node input sine wave signal was $200 \mathrm{~Hz}$, and the length of shielded twisted pair (STP) between nodes was $20 \mathrm{~m}$. Then using these two methods began the experiment and recorded their collection speed. Thirdly, the same experiments were done when the sampling rate was $25 \mathrm{KSPS}$ and $50 \mathrm{KSPS}$. Lastly, the number of connected slave nodes was changed to obtain the accurate results. Figure 7 recorded the relationship curve between collection speed and the quantity of slave nodes at different sampling rate.

As can be seen from the diagram, when sampling rates are the same, collection speed and the number of nodes are substantially linear $\left(V_{n}=n \times V_{1}, V_{1}\right.$ related to the sampling rate). When the sampling rate is $10 \mathrm{KSPS}, V_{1} \approx$ sampling rate $\times$ data block size $=10 \mathrm{KSPS} \times 2 \times 16 \mathrm{~B} / \mathrm{S}=0.32 \mathrm{MB} / \mathrm{s}$. Similarly, when the sampling rate is $25 \mathrm{KSPS}$ or $50 \mathrm{KSPS}$, acquisition rate is proportional to the number of slave nodes. And with the number of nodes increasing, the collection speed gradually increases. Because the STM through the slave 
node directly controls transmission process and saves the information transmission time between the master-slave nodes, STM is faster compared with ITM.

Experiment II. The master node connected 2 or 3 slave nodes and the twisted pair length between nodes was $20 \mathrm{~m}$. Every node's sampling rate was $50 \mathrm{KSPS}$, and input signal was $1 \mathrm{KHz}$ sine wave. Then using these two methods collected different data sizes, including $100 \mathrm{MB}, 200 \mathrm{MB}, 500 \mathrm{MB}, 1000 \mathrm{MB}$, $2000 \mathrm{MB}$ and $5000 \mathrm{MB}$. At last, using monitoring software analyzed the collected data and calculated bit error rate. Secondly, employing twisted pairs of $50 \mathrm{~m}$ and $100 \mathrm{~m}$, respectively, made similar experiments to verify the relationship between the collection size and transmission error rate at different length of twisted pair. All the measurement results are shown in Figure 8.

As shown in Figure 8, when the length of STP is fixed, error rate is reduced with the increase of the number of collection, and error rate of two slave nodes is lower than three slave nodes. When STP lengths were $30 \mathrm{~m}$ and $70 \mathrm{~m}$, the experiments were made by the same method. Due to the long-distance transmission attenuation of digital signal, the error rate increases as the length of the STP increases in the same data size collection, as shown in Figure 9. However, in the same condition, ITM's error rate is lower than the STM. Therefore, compared to STM, ITM has higher transmission reliability, better accuracy, and more expansion.

According to the state transition diagram, in the STM, the data of multiple nodes may exist on the bus at the same time, so there is interference during transmission. And the start delay of STM is smaller than the ITM. In the STM, a slave node by receiving the transfer instruction and the end flag in the previous node information realizes the start-up of this node transmission and suspends the transmission of the previous node. However, in ITM, the whole process is completed by the master node. Therefore, the pressure of slave nodes in STM is bigger than ITM, and the transmission control is more complex and extended performance is poorer.

Through the analysis and discussion of these test results, ITM has the characteristics of simple control, small transmission interference, low error rate, and fast collection speed, so it is suitable for large-scale factories, storehouses, and other occasions of distributed multiparameter measurement. Due to its excellent extensible performance and high reliability, it has widely application value in the industrial field.

\section{Conclusion}

According to the characteristics of the distributed parameter measurement application, a main structure of measurement system and the circuit design of the master-slave node are designed and introduced. Then this paper described a simple and feasible communication protocol and the basic control information and presented two control methods of distributed data acquisition and transmission. Thirdly, an experimental platform for testing experiments of the two methods is set up, and experimental results are also analyzed and discussed, including the acquisition rate, extendibility, and transmission reliability. Fourthly, through comparison with the previous method, the experiments verified the accuracy, the reliability, and the scalability of the ITM, which has the advantages of higher reliability, better extensible performance, and simpler control. Finally, the high-performance control method is applied to a project of multiarea environmental parameter measurement, which further verifies the correctness, feasibility, and reliability of the ITM.

\section{Conflict of Interests}

The authors declare that there is no conflict of interests regarding the publication of this paper.

\section{Acknowledgment}

This work was supported in part by the National Natural Science Foundation of China (no. 61335008).

\section{References}

[1] Z. Y. Nan and L. R. Hua, "Design and analysis for the wireless data acquisition network with star topological structure and low power consumption," Process Automation Instrumentation, vol. 32, no. 12, pp. 47-50, 2011.

[2] F. K. Hwang, "A complementary survey on double-loop networks," Theoretical Computer Science, vol. 263, no. 1-2, pp. 211229, 2001.

[3] C. K. Wong and D. Coppersmith, "A combinatorial problem related to multimodule memory organizations," Journal of the ACM, vol. 21, no. 3, pp. 392-402, 1974.

[4] J. H. Saltzer and K. T. Pogran, "A star-shaped ring network with high maintainability," Computer networks, vol. 4, no. 5, pp. 239244, 1979.

[5] B. W. Arden and H. Lee, "Analysis of chordal ring network," IEEE Transactions on Computers, vol. 30, no. 4, pp. 291-295, 1981.

[6] S. K. Chen, F. K. Hwang, and Y. C. Liu, "Some combinatorial properties of mixed chordal rings," Journal of Interconnection Networks, vol. 4, no. 1, pp. 3-16, 2003.

[7] C. Zhang, W. Ma, and C. Sun, "A switchable high-speed fiberoptic ring net topology and its method of high-performance synchronization for large-capacity power electronics system," International Journal of Electrical Power and Energy Systems, vol. 57, pp. 335-349, 2014.

[8] J. C. Bermond, F. Comellas, and D. F. Hsu, "Distributed loop computer-networks-a survey," Journal of Parallel and Distributed Computing, vol. 24, no. 1, pp. 2-10, 1995.

[9] L. Z. Hua, W. Z. Xin, and Z. H. Qiang, "Implementation of distributed wireless data acquisition system," Process Automation Instrumentation, vol. 31, no. 5, pp. 73-78, 2010.

[10] N. Chen, C. Xu, W. Zhuang, G. Chen, and Y. Li, "Distributed data acquisition of unified city and county dispatching automation system," Automation of Electric Power Systems, vol. 35, no. 24, pp. 89-92, 2011.

[11] A. Vasenev, T. Hartmann, and A. G. Dorée, "A distributed data collection and management framework for tracking construction operations," Advanced Engineering Informatics, vol. 28, no. 2, pp. 127-137, 2014.

[12] K. A. Corzine, S. Lu, and T. H. Fikse, "Distributed control of hybrid motor drives," IEEE Transactions on Power Electronics, vol. 21, no. 5, pp. 1374-1383, 2006. 
[13] Y. Peng, D.-F. Zhao, Z.-Z. Zhou, and G.-M. Zha, "Study on the application of distributed wireless token ring protocol in wireless ad hoc networks," Journal of Electronics and Information Technology, vol. 27, no. 4, pp. 629-633, 2005.

[14] M. Ergen, D. Lee, R. Sengupta, and P. Varaiya, "WTRPwireless token ring protocol," IEEE Transactions on Vehicular Technology, vol. 53, no. 6, pp. 1863-1881, 2004.

[15] Low-Voltage Differential Signaling (LVDS), Wikipedia, http:// en.wikipedia.org/wiki/Low-voltage_differential_signaling.

[16] F. Formenti and E. Scapparone, "Transmission of isolated LVDS signal pulses at long distance," Nuclear Instruments and Methods in Physics Research Section A: Accelerators, Spectrometers, Detectors and Associated Equipment, vol. 614, no. 2, pp. 319-322, 2010.

[17] EZ-USB Technical Reference Manual, http://www.cypress .com/.

[18] C. H. Wang and G. F. Jin, "The research and implementation of USB communication interface circuit based on FPGA," Applied Mechanics and Materials, vol. 220-223, pp. 2032-2035, 2012.

[19] J. S. Wu, F. Z. Shang, H. Sun, T. Qin, and Q. Li, "Design of data transmission system based on FPGA and USB 2.0," Applied Mechanics and Materials, vol. 321-324, pp. 417-422, 2013.

[20] S. Shujing and L. Jiansheng, "A method of multi-channel data acquisition with adjustable sampling rate," TELKOMNIKA Indonesian Journal of Electrical Engineering, vol. 11, no. 9, pp. 5299-5307, 2013.

[21] DS15BA101 1.5 Gbps Differential Buffer with Adjustable Output Voltage, 2013, http://www.ti.com.cn/cn/lit/ds/symlink/ds15ba101 .pdf.

[22] DS15EA101 0.15 to 1.5 Gbps Adaptive Cable Equalizer with LOS Detection, 2009, http://www.ti.com.cn/cn/lit/ds/symlink/ ds15ea101.pdf. 

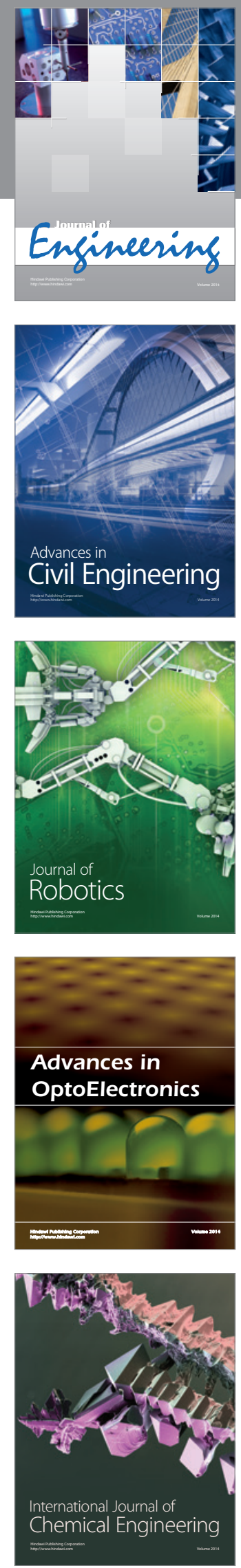

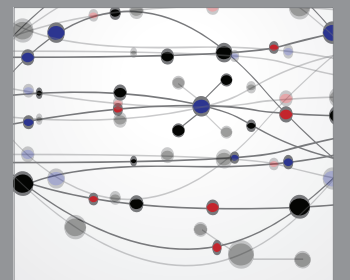

The Scientific World Journal
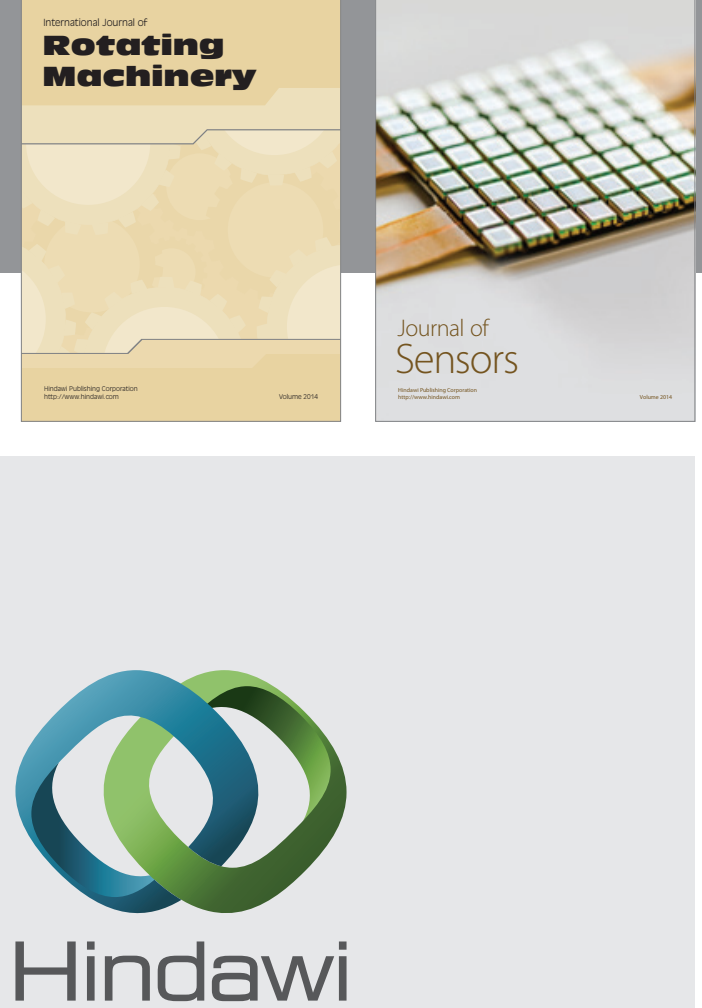

Submit your manuscripts at http://www.hindawi.com
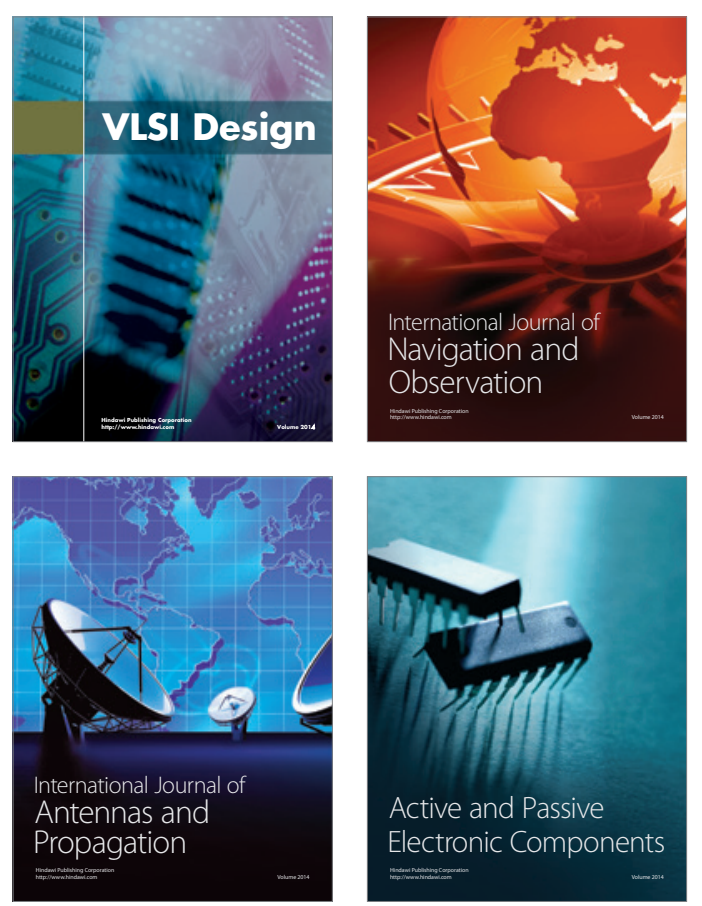
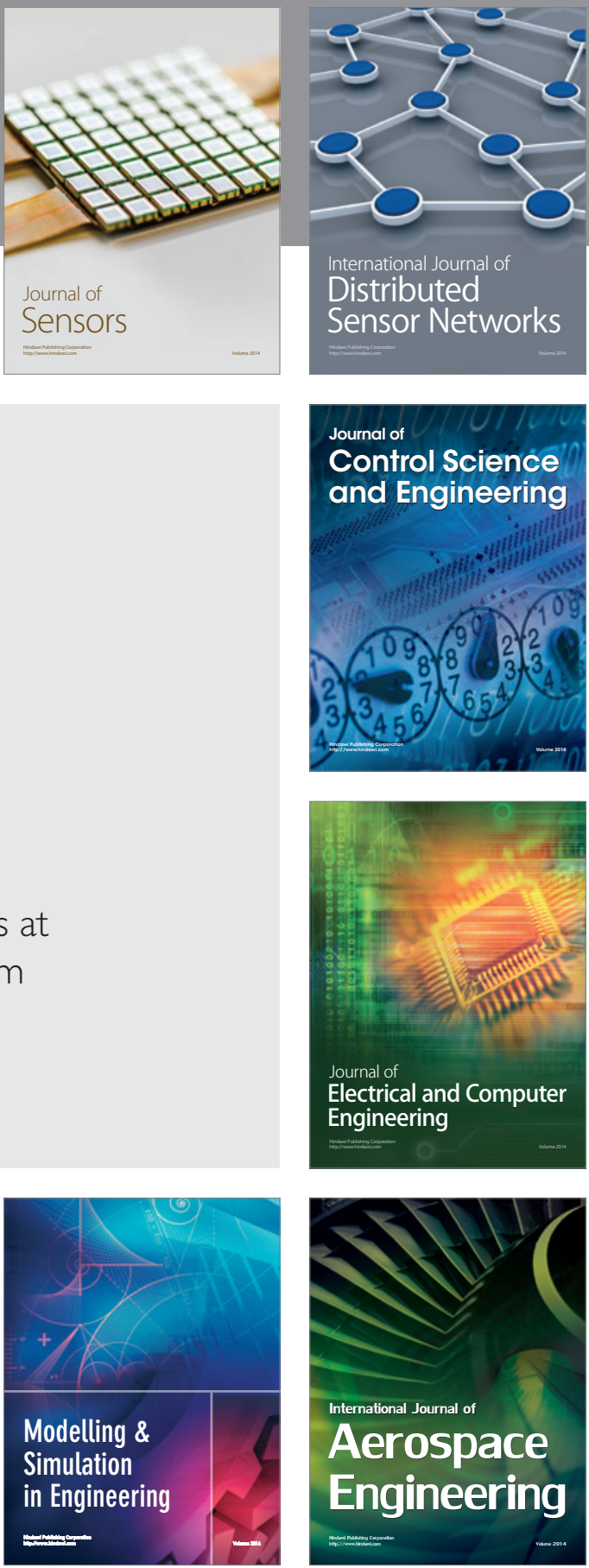

Journal of

Control Science

and Engineering
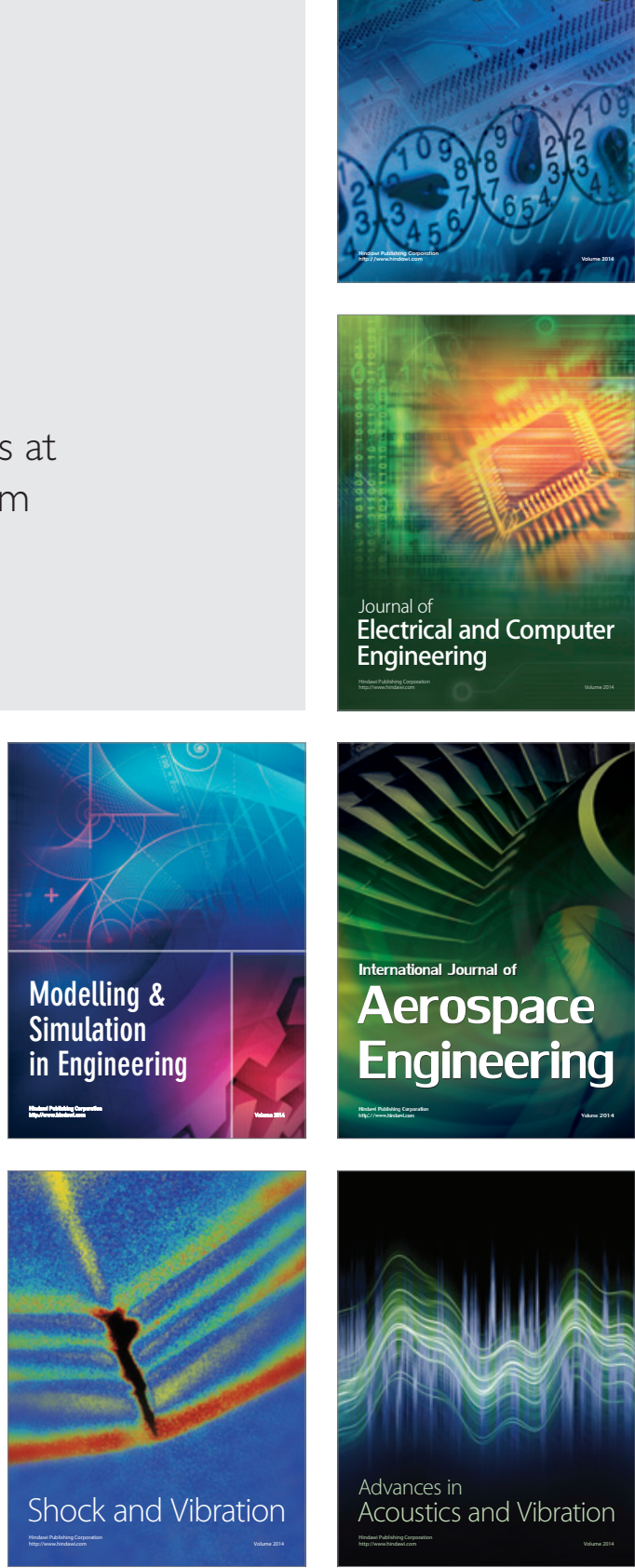